\title{
VECTOR COHERENT STATE THEORY FOR THE Sp(6) $\supset U(3)$ BRANCH OF THE S, D, FERMION PAIR ALGEBRA
}

\author{
K.T. HECHT ${ }^{4}$ \\ Physics Department, University of Michigan, Ann Arbor, Michigan 48109, USA \\ Received 25 January 1988
}

\begin{abstract}
Vector coherent state theory is used to give very explicit matrix representations for the $\mathrm{Sp}(6) \supset \mathrm{U}(3)$ branch of the Ginocchio S,D-pair algebra for all states of generalized S,D-pair seniority, $u=0,1$, and 2 to facilitate calculations in the fermion dynamic-symmetry model.
\end{abstract}

\section{Introduction}

Recently the Ginocchio S,D fermion pair algebra '), introduced originally as a toy model for the study of the fermionic foundation of the interacting boson model of Arima and lachello, has been proposed as a fermion dynamical-symmetry model meriting serious consideration for nuclear spectra throughout the periodic chart ${ }^{2-5}$ ). In this model the normal-parity single-particle states of the shell model are reclassified in terms of a pseudo-orbital angular momentum $(k)$ and a pseudo-spin (i), with $\boldsymbol{k}+\boldsymbol{i}=\boldsymbol{j}$. The so-called $i$-active version of the model with $i$ limited to $i=\frac{3}{2}$ leads to the $\mathrm{SO}(8)$ symmetry of Ginocchio. State constructions for the three subgroup chains of the $S O(8)$ symmetry have been worked out in considerable detail ${ }^{1,6.7}$ ). Recently, in particular, it has been shown ${ }^{7}$ ) that the new vector coherent state techniques ${ }^{8-12}$ ), [for a detailed list of applications see ref. $\left.{ }^{12}\right)$, can be exploited to give very explicit matrix representations for the physically interesting states of low (but nonzero) generalized S,D-pair seniority for both the $\mathrm{SO}(8) \supset \mathrm{SO}(6) \times \mathrm{SO}(2)$ and $\mathrm{SO}(7) \neg \mathrm{SO}(5) \times \mathrm{SO}(2)$ branches of the model. Matrix elements of fermion model hamiltonians, built from terms of the interacting boson type, can thus be evaluated directly, on an equal footing for both even and odd nuclei, as well as for the most interesting states of higher S,D-pair seniority.

Until recently the $k$-active version of the model with $k$ limited to $k=1$, leading to a $\mathrm{Sp}(6)$ symmetry with a $\mathrm{Sp}(6) \supset \mathrm{SU}(3)$ branch, has received less attention. This resulted from the "flaw" of the $S p(6)$ model, noted by Ginocchio, that it cannot support states of high $\mathrm{SU}(3)$ quantum numbers $(\lambda \mu)$ and thus does not appear to be a good candidate for nuclei with strongly collective quadrupole rotational spectra. In a $j=\frac{1}{2}, \frac{3}{2}, \frac{5}{2}, \frac{7}{2}, \frac{9}{2}$ shell, for example, the largest value of $\lambda+\mu$ permitted by the Pauli principle in this Sp(6) model, [with $i=\frac{1}{2}, \frac{7}{2} ; k=1$ ], is $\lambda+\mu=10,\left[\lambda+\mu \leqslant \frac{2}{3} \Omega\right.$, with $\left.\Omega=\sum_{j}\left(j+\frac{1}{2}\right)\right]$. The interacting boson model would permit states with $\lambda+\mu=14$ in even nuclei of such a shell, whereas the Elliott SU(3) model in configurations of

S Supported in part by the US National Science Foundation. 
identical nucleons of an sdg shell can support representations with $\lambda+\mu$ as high as $\left.{ }^{13}\right) \lambda+\mu=26$. The $\operatorname{Sp}(6, \mathrm{R})$ model of Rosensteel and Rowe $\left.{ }^{14}\right)$, on the other hand, appears to give a sound shell-model foundation of the nuclear collective model and lcads to the possibility of truly microscopic calculations of nuclear collective phenomena $\left.{ }^{15,16}\right)$. In the $\operatorname{Sp}(6, \mathrm{R}) \supset \mathrm{SU}(3)$ symmetry states of very large $(\lambda+\mu)$ are attained through a coupling of an intrinsic $\mathrm{SU}(3)$ representation with a collective SU(3) representation. An attempt to increase the possible $(\lambda+\mu)$ values of the Ginocchio $\mathrm{Sp}(6)$ model by a similar mechanism led to failure ${ }^{17}$ ). Although states with relatively large intrinsic $(\lambda \mu)$ representations, resulting from $\mathrm{Sp}(6)$ representations with higher generalized $S, D$-pair seniority quantum numbers, can in principle be coupled with the favored S,D-pair configurations of high $(\lambda \mu)$ to lead to states with a resultant high SU(3) symmetry, the limit $\lambda+\mu \leqslant \frac{2}{3} \Omega$ cannot be exceeded. Moreover, the combination of intrinsic and collective SU(3) symmetries now leads to the disappearance $\left.{ }^{17}\right)$ of states such as $(\lambda \mu)=\left(\frac{2}{3} \Omega-2,2\right)$ which are among the Pauli-allowed states in the $\mathrm{Sp}(6)$ representation corresponding to zero generalized S,D-pair seniority and intrinsic $(\lambda \mu)=(00)$.

Despite these difficulties the recent work on the fermion dynamic-symmetry model has led to renewed interest in the $S p(6) \supset S U(3)$ branch of the $S, D$ fermion pair algebra. States of low generalized S,D-pair seniority may be the most relevant, and it may again be useful to be able to treat even and odd nuclei as well as states of nonzero S,D-pair seniority on an equal footing. Vector coherent state techniques are again ideal for this purpose and require only a small change in the formulas valid for the noncompact counterpart, the $\operatorname{Sp}(6, R)$ algebra. One of the central features of the vector coherent state method is the associated $K$-matrix theory ${ }^{8}$ ), and it is the development of the $K$-matrix technique into a viable computational tool which has led to the many recent detailed applications ${ }^{12}$ ). It is the purpose of this short note to demonstrate that the $K$-matrices for the $\mathrm{Sp}(6) \supset \mathrm{SU}(3)$ model and states of generalized S,D-pair seniority $v=0,1$, and 2 can be given in completely analytic form and thus leads to complete and detailed constructions of the matrix representations of the physically relevant states of the $\operatorname{Sp}(6) \supset \mathrm{SU}(3)$ branch of the fermion dynamical-symmetry model. For the noncompact $\operatorname{Sp}(6, \mathrm{R}) \supset \mathrm{SU}(3)$ symmetry and nuclei with mass numbers $A>6$, Rowe, Wybourne, and Butler ${ }^{18}$ ) have shown that the $K^{2}$ matrices are completely free of zero eigenvalues. As a result all states of this symmetry are Pauli-allowed. In the $S p(6) \supset S U(3)$ model, on the other hand, the zero eigenvalues of the $K^{2}$ matrices play a prominent role and indicate very explicitly the Pauli-allowed domain of this model, another powerful reason for treating this problem by vector coherent state techniques.

\section{The vector coherent state theory of the $S p(6) \supset S U(3) S, D-p a i r$ model}

In the $\mathrm{S}, \mathrm{D}$, fermion pair model the single-nucleon operators, $a_{j m}^{\dagger}$, for a mixed configuration of $j$-values are given in terms of pseudo angular momenta $k$ and $i$ 
with $k+i=j:$

$$
a_{j m}^{\dagger}=\sum_{m_{k}, m_{i}}\left\langle k m_{k} i m_{i} \mid j m\right\rangle b_{k m_{k}, i m_{i}}^{\dagger} .
$$

In the $\mathrm{Sp}(6)$ model $k$ is restricted to $k=1$, so that with $i=\frac{1}{2}, \frac{7}{2}$ we can build a $j=\frac{1}{2}, \frac{3}{2}, \frac{5}{2}, \frac{7}{2}, \frac{9}{2}$ shell, for example. With $i=\frac{3}{2}$ and $\frac{9}{2}$ this is a model for a $j=\frac{1}{2}, \frac{3}{2}, \frac{5}{2}, \frac{7}{2}$, $\frac{9}{2}, \frac{11}{2}$ shell. The $\operatorname{Sp}(6)$ algebra is generated by the pair creation operators, $A_{I M}^{\dagger}$, (with 2-particle pseudo-spin angular momentum $I=0$ ), the hermitian conjugate pair annihilation operators, $A_{J M}$, and the nine $I=0$ one-body operators which generate the $\mathrm{U}(3)$ subalgebra. In the normalization of refs. $\left.{ }^{4,6}\right)$, with $\Omega_{k i}=\frac{1}{2}(2 k+1)(2 i+1)$, and $k=1$ :

$$
A_{J M}^{\dagger}=\sum_{i} \sqrt{\frac{1}{2} \Omega_{k i}}\left[b_{k i}^{\dagger} \times b_{k i}^{\dagger}\right]_{M}^{J} \stackrel{0}{0}, \quad J=0,2 .
$$

In order to parallel the vector coherent state construction $\left.{ }^{8}\right)$ of the $\mathrm{Sp}(6, \mathrm{R})$ algebra as closely as possible it is convenient to introduce cartesian components

$$
A_{a b}^{\dagger}=A_{b a}^{\dagger}=\sum_{i, m_{i}}(-1)^{i-m_{i}} b_{x_{a} i m_{i}}^{\dagger} b_{x_{b} i-m_{i}}^{\dagger}
$$

where

$$
b_{1 \pm 1 i m_{i}}^{\dagger}=\mp \sqrt{\frac{1}{2}}\left(b_{x i m_{i}}^{\dagger} \pm i b_{y i m_{i}}^{\dagger}\right), \quad b_{10 i m_{i}}^{\dagger}=b_{z i m_{i}}^{\dagger} .
$$

Similarly, the one-body operators in cartesian form are

$$
C_{a b}=\left(\sum_{i, m_{i}} b_{x_{a} i m_{i}}^{+} b_{x_{b} i m_{i}}\right)-\frac{1}{3} \delta_{a b} \Omega,
$$

where $\Omega=3 \sum_{i}\left(i+\frac{1}{2}\right)=\sum_{j}\left(j+\frac{1}{2}\right)$. The $A_{J M}^{*}$ of eq. (2) are given in terms of the cartesian components by

$$
\begin{aligned}
A_{2 \pm 2}^{\dagger} & =\frac{1}{4} \sqrt{3}\left(A_{x x}^{\dagger}-A_{y y}^{\dagger} \pm 2 i A_{x y}^{\dagger}\right), \\
A_{2 \pm 1}^{\dagger} & =\mp \frac{1}{2} \sqrt{3}\left(A_{x z}^{\dagger} \pm i A_{y z}^{+}\right), \\
A_{20}^{+} & =\frac{1}{2} \sqrt{\frac{1}{2}}\left(-A_{x x}^{+}-A_{y y}^{\dagger}+2 A_{z z}^{\dagger}\right), \\
A_{00}^{\dagger} & =-\frac{1}{2}\left(A_{x x}^{\dagger}+A_{y y}^{\dagger}+A_{z z}^{\dagger}\right) .
\end{aligned}
$$

Wich $B_{a b} \equiv A_{a b}=\left(A_{a b}^{\dagger}\right)^{\dagger}$, the commutator algebra for the $\operatorname{Sp}(6)$ generators is

$$
\begin{aligned}
& {\left[A_{a b}^{\dagger}, A_{c d}\right]=\delta_{a c} C_{b d}+\delta_{b d} C_{a c}+\delta_{b c} C_{a d}+\delta_{a d} C_{b c},} \\
& {\left[A_{a b}, C_{c d}\right]=\delta_{a c} A_{b d}+\delta_{b c} A_{a d},} \\
& {\left[C_{a b}, C_{c d}\right]=\delta_{b c} C_{a d}-\delta_{a d} C_{c b},}
\end{aligned}
$$

which, with the exception of a few crucial changes in sign, is identical with that of the noncompact $\mathrm{Sp}(6, \mathrm{R})$ algebra. In analogy with ref. $\left.{ }^{8}\right)$ the vector coherent state for the $S p(6) \supset \mathrm{U}(3)$ algebra can then be built in terms of six complex variables $z_{a b}$ $\left(=z_{b a}\right)$

$$
|z\rangle=\exp \left(\frac{1}{2} \sum_{a, b} z_{a h}^{*} A_{a h}^{\dagger}\right)|[\sigma] \alpha\rangle,
$$


where $[\sigma] \equiv\left[\sigma_{1} \sigma_{2} \sigma_{3}\right]$ is the "intrinsic" $U(3)$ symmetry, i.e. the $U(3)$ symmetry of the $u$ nucleons entirely free of the favored S,D pairs of the model, so that

$$
A_{a b}|[\sigma] \alpha\rangle=0 \quad \text { for all } a, b ; \alpha,
$$

where $\alpha$ is a shorthand label for any convenient set of $\mathrm{U}(3)$ subgroup labels, and $u=\sigma_{1}+\sigma_{2}+\sigma_{3}$ is the generalized S,D-pair seniority number. Since the Cartan subalgebra is generated by the three $C_{a a}$ and the highest weight values of these is given by $\frac{1}{3} \Omega-\sigma_{3}, \frac{1}{3} \Omega-\sigma_{2}, \frac{1}{3} \Omega-\sigma_{1}$, the $\operatorname{Sp}(6)$ irreducible representation is labeled by the $\sigma_{i}$ and, in Cartan standard form, is given by

$$
\left(\frac{1}{3} \Omega-\sigma_{3}, \frac{1}{3} \Omega-\sigma_{2}, \frac{1}{3} \Omega-\sigma_{1}\right) .
$$

State vectors are mapped into $z$-space functional representations ${ }^{8,12}$ )

$$
|\psi\rangle \rightarrow \psi_{[\sigma] \alpha}(z)=\langle z \mid \psi\rangle=\langle[\sigma] \alpha| \mathrm{e}^{\frac{1}{2 \sum_{u, h} z_{u h} A_{u h}}|\psi\rangle}
$$

and, operators $\boldsymbol{O}$ are mapped into their $\boldsymbol{z}$-space realizations, $\Gamma(\boldsymbol{O})$,

$$
\boldsymbol{O}|\psi\rangle \rightarrow \Gamma(\boldsymbol{O}) \psi_{[\sigma] \alpha}(z)
$$

Defining $\nabla_{a b}=\left(1+\delta_{a b}\right) \partial / \partial z_{a b}$, the $z$-space realizations of the $21 \operatorname{Sp}(6)$ generators are given by

$$
\begin{aligned}
& \Gamma\left(A_{a b}\right)=\nabla_{a b}, \\
& \Gamma\left(C_{a b}\right)=\mathbb{C}_{a b}+z_{a \beta} \nabla_{\beta b}=\mathbb{C}_{a b}^{\mathrm{intr}}+C_{a b}^{\mathrm{coll},}, \\
& \Gamma\left(A_{a b}^{+}\right)=-\mathbb{C}_{a \beta} z_{\beta b}-\mathbb{C}_{b \beta} z_{\beta a}-z_{a \beta} z_{b \gamma} \nabla_{\beta \gamma} .
\end{aligned}
$$

Summation convention is used for repeated (Greek) indices. Note the change of sign from the corresponding $z$-space realizations of $\operatorname{Sp}(6, \mathrm{R})$, (cf. refs. ${ }^{8,12}$ )]. The $\mathbb{C}_{a b}$ are the "intrinsic" U(3) generators which act only on the intrinsic states $|[\sigma] \alpha\rangle$, i.e. the states of generalized S,D-pair seniority $u=\sigma_{1}+\sigma_{2}+\sigma_{3}$, the vector "vacuum" states of the $\mathrm{Sp}(6)$ symmetry which are annihilated by the favored $\mathrm{S}, \mathrm{D}$-pair annihilation operators, $A_{J M}$. The $\mathbb{C}_{a b}$ commute with the $z_{c d}$ and $\nabla_{c d}$, so that the $\operatorname{Sp}(6)$ algebra has been mapped into a direct sum of a 6-dimensional oscillator (Heisenberg-Weyl) algebra, generated by the $z_{c d}$ and $\nabla_{c d}$, and an intrinsic U(3) algebra generated by the $\mathbb{C}_{a b}$. The 6-dimensional oscillator algebra generated by $z$-space polynomials in the 6-dimensional space of the $z_{a b}$ must be reduced with respect to $\mathrm{U}(3)$. In the 6-dimensional Bargmann space of the $z_{a b}$, the oscillator functions are constructed through polynomials of degree $n_{1}+n_{2}+n_{3}, Z^{\left[n_{1} n_{2} n_{3}\right]}(z)$, carrying the "collective" $\mathrm{U}(3)$ quantum numbers $\left[n_{1} n_{2} n_{3}\right]$ with $n_{i}$ all even integers, which can then be coupled to the "intrinsic" $\mathrm{U}(3)$ quantum numbers $\left[\sigma_{1} \sigma_{2} \sigma_{3}\right]$

$$
\left[Z^{\left[n_{1} n_{2} n_{3}\right]}(z) \times\left|\left[\sigma_{1} \sigma_{2} \sigma_{3}\right]\right\rangle\right]_{\eta}^{\left[\omega_{1} \omega_{2} \omega_{3}\right] p},
$$

where the square bracket denotes the $U(3)$ coupling $\left[\left[\sigma_{1} \sigma_{2} \sigma_{3}\right] \times\left[n_{1} n_{2} n_{3}\right]\right] \rightarrow\left[\omega_{1} \omega_{2} \omega_{3}\right]$ to states of resultant $U(3)$ symmetry specified by $\left[\omega_{1} \omega_{2} \omega_{3}\right] \equiv[\omega]$. The multiplicity label, $\rho$, is needed if the $\mathrm{U}(3)$ coupling $[[\sigma] \times[n]]$ contains the resultant $\mathrm{U}(3)$ 
representation $[\omega]$ with a multiplicity greater than one. The labels $\eta$ can be any convenient set of U(3) subgroup labels. (Note also that we adhere to a right to left U(3) coupling order in eq. (13)).

The states of eq. (13) form an orthonormal set with respect to a $z$-space scalar product with a Bargmann measure. Since the $z$-space realizations $\Gamma\left(A_{a b}\right), \Gamma\left(A_{a b}^{\ddagger}\right)$ of eq. (12) are not unitary with respect to this measure, the $z$-space realizations, $\Gamma\left(A_{a b}\right), \Gamma\left(A_{a b}^{+}\right)$must still be transformed to unitary form, $\gamma\left(A_{a b}\right), \gamma\left(A_{a b}^{\dagger}\right)$, via the $K$-operator ${ }^{8,12}$ )

$$
\gamma(\boldsymbol{A})=K^{-1} \Gamma(\boldsymbol{A}) K, \quad \gamma\left(\boldsymbol{A}^{\dagger}\right)=K^{-1} \Gamma\left(\boldsymbol{A}^{\dagger}\right) K,
$$

where $K$ is a $\mathrm{U}(3)$ scalar since it commutes with $\Gamma(C)=\gamma(C)$. The unitary requirement $\gamma\left(A_{a b}^{\dagger}\right)=\left(\gamma\left(A_{a b}\right)\right)^{\dagger}=K^{\dagger} z_{a b}\left(K^{-1}\right)^{\dagger}$ leads to

$$
\Gamma\left(A_{a b}^{\dagger}\right) K K^{\dagger}=K K^{\dagger} z_{a b} \text {. }
$$

This will be solved for $K K^{\dagger}$ through the introduction of the auxiliary "Toronto operator," $A_{\mathrm{op}}$, with the property

$$
\left[A_{o p,}, z_{a b}\right]=\Gamma\left(A_{a b}^{\dot{i}}\right)
$$

and the solution

$$
\begin{aligned}
A_{\mathrm{op}}= & -\frac{1}{2}\left(\mathcal{C}_{\alpha \beta}+z_{\alpha \alpha \beta} \nabla_{\alpha \beta \beta}\right)\left(\mathcal{C}_{\beta \alpha}+z_{\beta \mu} \nabla_{\mu \alpha \alpha}\right) \\
& +\frac{1}{4} z_{\alpha x \nu} \nabla_{\psi \beta} z_{\beta \mu \alpha} \nabla_{\mu \alpha}+z_{\alpha \beta} \nabla_{\beta \alpha} \\
= & -\frac{1}{2} C_{\alpha \beta}^{\mathrm{rot}} C_{\beta \alpha}^{\mathrm{tot}}+\frac{1}{4} C_{\alpha \beta}^{\mathrm{coll}} C_{\beta \alpha}^{\mathrm{coll}}+z_{\alpha \beta} \nabla_{\beta \alpha},
\end{aligned}
$$

with eigenvalue given by the $\mathrm{U}(3)$ Casimir invariants $C_{\alpha \beta}^{\text {tot }} C_{\beta \alpha}^{\text {tat }}$ and $C_{\alpha \beta}^{\text {coll }} C_{\beta \alpha \beta}^{\text {coll }}$, with $C_{a b}^{\text {tot }}=\mathbb{C}_{a b}^{\mathrm{intr}}+C_{a b}^{\mathrm{coll}}$.

$$
\begin{aligned}
A_{\text {eigen }}= & -\frac{1}{2}\left[h^{2}\left(\omega_{1}\right)+h^{2}\left(\omega_{2}\right)+h^{2}\left(\omega_{3}\right)+2 h\left(\omega_{1}\right)-2 h\left(\omega_{3}\right)\right] \\
& +\frac{1}{4}\left[n_{1}^{2}+n_{2}^{2}+n_{3}^{2}+2 n_{1}-2 n_{3}\right]+n_{1}+n_{2}+n_{3},
\end{aligned}
$$

where

$$
h\left(\omega_{i}\right)=\omega_{i}-\frac{1}{3} \Omega, \quad h\left(n_{i}\right)=n_{i}, \quad h\left(\sigma_{i}\right)=\sigma_{i}-\frac{1}{3} \Omega .
$$

(Note that the dependence on the $\sigma_{i}$ is implicit only, through the coupling $[\sigma] \times[n] \rightarrow$ $[\omega]$.)

The important quantities are the eigenvalue differences

$$
\begin{aligned}
A_{\left[n^{\prime}\right]\left[\omega^{\prime}\right]}-A_{[n][\omega]}= & -\frac{1}{2}\left(\Delta \omega_{1}^{2}+\Delta \omega_{2}^{2}+\Delta \omega_{3}^{2}\right)-\left(\omega_{1}+1\right) \Delta \omega_{1}-\omega_{2} \Delta \omega_{2} \\
& -\left(\omega_{3}-1\right) \Delta \omega_{3}+\frac{2}{3} \Omega+n_{i}+5-i,
\end{aligned}
$$

where the $\mathrm{U}(3)$ coupling $\left[\omega_{1} \omega_{2} \omega_{3}\right] \times[2] \rightarrow\left[\omega_{1}^{\prime} \omega_{2}^{\prime} \omega_{3}^{\prime}\right]$ leads to the two possibilities $\Delta \omega_{j}=2$ or $\Delta \omega_{i}=1, \Delta \omega_{k}=1$; but $\Delta n_{i}=2$ only for the coupling $\left[n_{1} n_{2} n_{3}\right] \times[2] \rightarrow$ $\left[n_{1}^{\prime} n_{2}^{\prime} n_{3}^{\prime}\right]$, and the index $i$ in eq. (20) gives the row to which the 2 squares are added 
in this product. (Note also the difference in signs from the corresponding expression for $\operatorname{Sp}(6, \mathrm{R})$, cf. $\left.\operatorname{refs}^{8,12}\right)$.)

The combination of eqs. (15) and (16) leads to the determination of $K K^{\dagger}$ through

$$
\left(\Lambda_{\mathrm{op} .} z_{a b}-z_{a b} \Lambda_{\mathrm{op}}\right) K K^{\dagger}=K K^{\dagger} z_{a b}
$$

Taking matrix elements between states $|[[\sigma] \times[\boldsymbol{n}]][\omega] \rho ; \eta\rangle[\mathrm{cf}$. eq. (13)], on the right and a similar state $\left\langle\left[[\sigma] \times\left[n^{\prime}\right]\right]\left[\omega^{\prime}\right] \rho^{\prime} ; \eta^{\prime}\right|$ on the left, this leads to an equation for the recursive determination of the $K K^{\dagger}$ matrix elements. After factoring out a common SU(3) Wigner coefficient this leads to

$$
\begin{gathered}
\sum_{[\bar{n}] \bar{\rho}}\left(A_{\left[n^{\prime}\right]\left[\omega^{\prime}\right]}-\Lambda_{[\bar{n}][\omega]}\right)\left\langle\left[[\sigma] \times\left[n^{\prime}\right]\right]\left[\omega^{\prime}\right] \rho^{\prime}\|z\|[[\sigma] \times[\bar{n}]][\omega] \bar{\rho}\right\rangle\left(K K^{\dagger}([\omega])\right)_{[\bar{n}] \bar{\rho},[n] \rho} \\
\quad=\sum_{\left[\bar{n}^{\prime}\right] \bar{\rho}^{\prime}}\left(K K^{\dagger}\left(\left[\omega^{\prime}\right]\right)\right)_{\left[n^{\prime}\right] \rho^{\prime},\left[\bar{n}^{\prime}\right] \bar{\rho}^{\prime}}\left\langle\left[[\sigma] \times\left[\bar{n}^{\prime}\right]\right]\left[\omega^{\prime}\right] \bar{\rho}^{\prime}\|z\|[[\sigma] \times[n]][\omega] \rho\right\rangle
\end{gathered}
$$

where the SU(3) reduced matrix elements of $z$ are given by

$$
\begin{aligned}
\langle[[\sigma] & \left.\left.\times\left[n^{\prime}\right]\right]\left[\omega^{\prime}\right] \rho^{\prime}\|z\|[[\sigma] \times[n]][\omega] \rho\right\rangle \\
& =U\left([\sigma][n]\left[\omega^{\prime}\right][2] ;[\omega] \rho_{-} ;\left[n^{\prime}\right] \rho_{-}^{\prime}\right)\left\langle\left[n^{\prime}\right]\|z\|[n]\right\rangle .
\end{aligned}
$$

The $U$-coefficient is a $U(3)$, (or equivalent $S U(3)$ ), Racah coefficient in unitary form which is readily available through the code of Draayer and Akiyama ${ }^{19}$ ). When not needed the multiplicity labels are replaced by a dash or omitted altogether. The $\mathrm{SU}(3)$ reduced matrix elements of the 6-dimensional $z$ in the pure collective subspace $\left\langle\left[\boldsymbol{n}^{\prime}\right]\|\boldsymbol{z}\|[\boldsymbol{n}]\right\rangle$ are given through eq. (2.25) of ref. ${ }^{8}$ ). (The reduced matrix elements of eq. (2.25) of ref. ${ }^{8}$ ) are to be multiplied with an additional $\sqrt{\frac{1}{2}}$ factor to adhere to later normalizations; see p. 84 of ref. ${ }^{12}$ ).) The $K K^{\dagger}$ submatrices, diagonal in [ $\left.\omega\right]$, are labeled by row/column indices [n] $\rho$. In many of the states of low gencralized S,D-pair seniority, multiplicity labels $\rho$ are not needed and [n] is uniquely determined by $[\sigma]$ and $[\omega]$ so that the $K K^{\dagger}$ submatrix collapses to a simple $1 \times 1$ matrix. If both states [ $\left.\omega^{\prime}\right]$ and $[\omega]$ fall into the 1-dimensional category, the reduced matrix elements of $z$ drop out of eq. (22), and this equation collapses to a simple recursion formula

$$
\frac{\left(K^{2}\left(\left[\omega^{\prime}\right]\right)\right)_{\left[n^{\prime}\right]\left[n^{\prime}\right]}}{\left(K^{2}([\omega])\right)_{[n][n]}}=\Lambda_{\left[n^{\prime}\right]\left[\omega^{\prime}\right]}-\Lambda_{[n][\omega]} .
$$

Note that in this case $K=K^{\dagger}$ and we have given $K K^{\dagger}$ its more conventional ${ }^{8,12}$ ) name $K^{2}$.

In the general case, inversion of the relation $\gamma\left(A_{a b}^{\dagger}\right)=K^{\dagger} z_{a b}\left(K^{-1}\right)^{\dagger}$ transforms the orthonormal Bargmann-space vectors of eq. (13) into the orthonormal base vectors

$$
|[\sigma][\omega] i ; \eta\rangle=\sum_{[n] \rho}\left(K^{-1}([\omega])\right)_{i .[n] o}\left[Z^{[n]}\left(A^{\dagger}\right) \times|[\sigma]\rangle\right]_{n}^{[\omega] \rho}
$$


expressed in ordinary space. The $z$ in $Z^{[n]}(z)$ are replaced by the pair creation operators $\boldsymbol{A}^{\dagger}$ in these polynomials. This involves the conversion of the $K K^{\dagger}$ matrices to the $K$ and $K^{-1}$ form. If $K K^{\dagger}$ is diagonalized by the unitary matrix, $U$,

$$
U\left(K K^{\dagger}\right) U^{*}=\lambda
$$

with $\lambda_{i j}=\delta_{i j} \lambda_{i}$ the inverse of the $K$-matrix can be chosen as

$$
\left(K^{-1}\right)_{i,[n] \rho}=\lambda_{i}^{-1 / 2} U_{i,[n] \rho},
$$

where $\lambda_{i}$ is one of the nonzero eigenvalues of $K K^{*}$. Similarly

$$
(K)_{[n\}_{p, i} i}=U_{[n]_{p, i} \lambda_{i}^{1 / 2}}^{*} \text {. }
$$

In the present application the $\left(K K^{\dagger}\right)$ are not only hermitian but real symmetric matrices, leading to real $U$. In eq. (25) we have converted $\left(K^{-1}\right)^{\dagger}=U_{[n] p, i}^{\dagger} \lambda_{i}^{-1 / 2}$ to $\lambda_{i}^{-1 / 2} U_{i,[n] \rho}=K^{-1}$. In the general case, eq. (25) leads to the SU(3)-reduced matrix element of the pair creation operators, $\boldsymbol{A}^{*}$,

$$
\begin{aligned}
\left\langle[\sigma]\left[\omega^{\prime}\right] j^{\prime}\left\|A^{\prime}\right\|[\sigma][\omega] i\right\rangle= & \sum_{[\boldsymbol{n}] \rho} \sum_{\left[\boldsymbol{n}^{\prime}\right] \rho^{\prime}}\left(\boldsymbol{K}^{-1}([\omega])\right)_{i,[\boldsymbol{n}] \rho}\left(K\left(\left[\omega^{\prime}\right]\right)\right)_{\left[\boldsymbol{n}^{\prime}\right] \rho^{\prime}, \boldsymbol{j}^{\prime}} \\
& \times U\left([\sigma][\boldsymbol{n}]\left[\boldsymbol{\omega}^{\prime}\right][2] ;[\omega] \rho_{-} ;\left[\boldsymbol{n}^{\prime}\right]_{-} \rho^{\prime}\right)\left\langle\left[\boldsymbol{n}^{\prime}\right]\|\boldsymbol{z}\|[\boldsymbol{n}]\right\rangle .
\end{aligned}
$$

In the special case when both initial and final state are simple, with 1-dimensional $K K^{+}$submatrices for which $[n]$ is uniquely determined by $[\sigma]$ and $[\omega]$ and $\rho$ is not needed, this collapses to the simpler formula

$$
\begin{aligned}
\left\langle[\sigma]\left[\omega^{\prime}\right]\left\|A^{+}\right\|[\sigma][\omega]\right\rangle= & {\left[\left(\Lambda_{\left[n^{\prime}\right]\left[\omega^{\prime}\right]}-\Lambda_{[n][\omega]}\right)\right]^{1 / 2}\left\langle\left[n^{\prime}\right]\|z\|[n]\right\rangle } \\
& \times U\left([\sigma][n]\left[\omega^{\prime}\right][2] ;[\omega] ;\left[n^{\prime}\right]\right) .
\end{aligned}
$$

Note that for the $A_{M}$ defined according to the normalizations of refs. ${ }^{4,6}$ ), as exhibited in eq. (2), the SU(3) reduced matrix elements of eqs. (29) and (30) must be multiplied by an additional factor of $\sqrt{\frac{3}{2}}$.

The SU(3) reduced matrix elements of the S,D pair annihilation operators follow from hermitian conjugation

$$
\left\langle[\sigma][\omega] i\|\boldsymbol{A}\|[\sigma]\left[\omega^{\prime}\right] j^{\prime}\right\rangle=\sqrt{\frac{\operatorname{dim}\left[\omega^{\prime}\right]}{\operatorname{dim}[\omega]}}(-1)^{\omega_{1}-\omega_{3}-\omega_{i}^{\prime}+\omega^{\prime}}\left\langle[\sigma]\left[\omega^{\prime}\right] j^{\prime}\left\|\boldsymbol{A}^{\dagger}\right\|[\sigma][\omega] i\right\rangle .
$$

\section{States with generalized S,D-pair seniority $u=0,1,2$}

If the favored S,D-pair excitations of the $S p(6) \supset U(3)$ model dominate the low-energy spectra of a nucleus, states of low generalized S,D-pair seniority may be of greatest relevance. However, it will be useful to be able to treat even and odd nuclei on an equal footing and to be able to examine states with nonzero generalized seniority, $u$. States with $u=0,1$, and 2 may be the most important. For these the 
$K K^{\dagger}$ matrices can be given in analytic form and in complete generality. For $u=0$ $\left[\sigma_{1} \sigma_{2} \sigma_{3}\right]=[000],\left(\lambda_{\sigma} \mu_{\sigma}\right)=(00)$ using Elliott SU(3) notation. For $u=1\left[\sigma_{1} \sigma_{2} \sigma_{3}\right]=$ $[100],\left(\lambda_{\sigma} \mu_{\sigma}\right)=(10)$. For $u=2$ there are two possibilities: $\left[\sigma_{1} \sigma_{2} \sigma_{3}\right]=[200],\left(\lambda_{\sigma} \mu_{\sigma}\right)=$ (20); or $\left[\sigma_{1} \sigma_{2} \sigma_{3}\right]=[110],\left(\lambda_{\sigma} \mu_{\sigma}\right)=(01)$. Since all of these have either $\lambda_{\sigma}-0$ or $\mu_{\sigma}=0$, no multiplicity labels are needed for the couplings $[\sigma] \times[n]$, and the Racah coefficients of eq. (23) have a particularly simple form. Tables $1-4$ give all possible $(\lambda \mu)=\left(\omega_{1}-\omega_{2}, \omega_{2}-\omega_{3}\right)$ for all particle numbers for these four cases for a $j=$ $\frac{1}{2} \frac{3}{2} \frac{79}{2} 2^{-}$-shell, with $\Omega=15$. The case $u=0$ has recently also been discussed by means

TABLE 1

Possible $(\lambda \mu)$ for the $u=0$ states of a $j=\frac{1}{2} \frac{3}{2} \frac{3}{2} \frac{79}{2}$ shell, $(\Omega=15)$. Sp(6) irrep (555).

\begin{tabular}{c|cccccccc}
\hline$n$ & \multicolumn{7}{|c}{$(\lambda \mu)$} \\
\hline 30 & $(00)$ & & & & & & \\
28 & $(02)$ & & & & & & & \\
26 & $(04)$ & $(20)$ & & & & & & \\
24 & $(06)$ & $(22)$ & $(00)$ & & & & & \\
22 & $(08)$ & $(24)$ & $(40)$ & $(02)$ & & & \\
20 & $(0,10)$ & $(26)$ & $(42)$ & $(04)$ & $(20)$ & & $(00)$ & \\
18 & & $(28)$ & $(44)$ & $(60)$ & $(06)$ & $(22)$ & $(02)$ \\
16 & & & $(46)$ & $(62)$ & $(08)$ & $(24)$ & $(40)$ & $(20)$ \\
14 & & & $(64)$ & $(26)$ & $(80)$ & $(42)$ & $(04)$ & $(20)$ \\
12 & & $(82)$ & $(44)$ & $(06)$ & $(60)$ & $(22)$ & $(00)$ & \\
10 & $(10,0)$ & $(62)$ & $(24)$ & $(40)$ & $(02)$ & & & \\
8 & $(80)$ & $(42)$ & $(04)$ & $(20)$ & & & & \\
6 & $(60)$ & $(22)$ & $(00)$ & & & & & \\
4 & $(40)$ & $(02)$ & & & & & & \\
2 & $(20)$ & & & & & & & \\
0 & $(00)$ & & & & & & & \\
\hline
\end{tabular}

TABLE 2

Possible $(\lambda \mu)$ for the $u=1$ states of a $j=\frac{1}{2} \frac{3}{2} \frac{5}{2} \frac{79}{2}$ shell, $(\Omega=15)$. Sp(6) irrep (554)

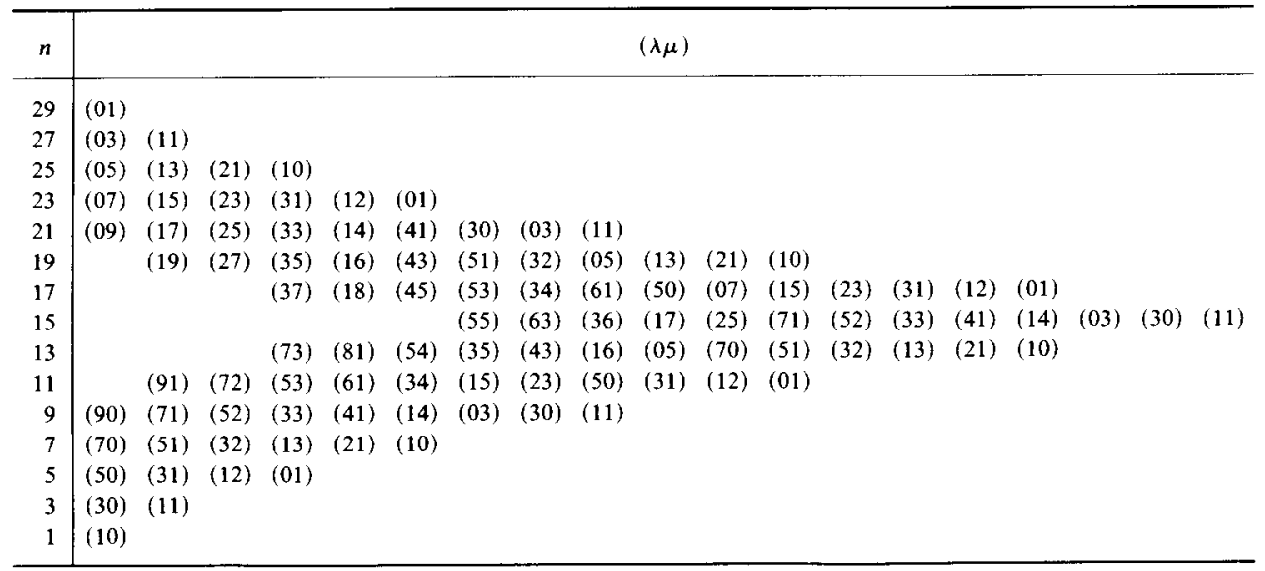


TABLE 3

Possible $(\lambda \mu)$ for the $u=2$ states, $\left(\lambda_{\sigma} \mu_{\alpha}\right)=(01)$, of a $j=\frac{1}{2} \frac{3}{2} \frac{5}{2} \frac{7}{2}$ shell, $(\Omega=15)$. Sp(6) irrep (544)

\begin{tabular}{|c|c|c|c|c|c|c|c|c|c|c|c|c|c|c|c|c|}
\hline$n$ & \multicolumn{16}{|c|}{$(\lambda \mu)$} \\
\hline 28 & (10) & & & & & & & & & & & & & & & \\
\hline 26 & (12) & (01) & & & & & & & & & & & & & & \\
\hline 24 & (14) & (03) & (30) & (11) & & & & & & & & & & & & \\
\hline 22 & (16) & (05) & (32) & (13) & (21) & (10) & & & & & & & & & & \\
\hline 20 & (18) & (07) & (34) & (15) & (23) & $(50)$ & $(31)$ & (12) & $(01)$ & & & & & & & \\
\hline 18 & & $(09)$ & (36) & (17) & (25) & $(52)$ & (33) & (41) & (14) & $(03)$ & 130 & (11) & & & & \\
\hline 16 & & & & & (27) & (54) & (35) & (43) & (70) & (51) & $(16$ & $(05)$ & (32) & (13) & (21) & (10) \\
\hline 14 & & & & & (72) & (45) & (53) & (34) & $(07)$ & (15) & $(61)$ & $(50)$ & (23) & (31) & (12) & $(01)$ \\
\hline 12 & & (90) & (63) & (71) & (52) & (25) & (33) & (14) & (41) & $(30)$ & $(03$ & (11) & & & & \\
\hline 10 & $(81)$ & (70) & (43) & (51) & (32) & (05) & (13) & (21) & (10) & & & & & & & \\
\hline 8 & (61) & $(50)$ & (23) & (31) & (12) & $(01)$ & & & & & & & & & & \\
\hline 6 & (41) & (30) & (03) & (11) & & & & & & & & & & & & \\
\hline 4 & (21) & (10) & & & & & & & & & & & & & & \\
\hline 2 & (01) & & & & & & & & & & & & & & & \\
\hline
\end{tabular}

TABLE 4

Possible $(\lambda \mu)$ for the $u=2$ states, $\left(\lambda_{t r} \mu_{t r}\right)=(20)$, of a $j=\frac{1}{2} \frac{3}{2} \frac{5}{2} \frac{79}{2}$ shell, $(\Omega=15)$. Sp(6) irrep (553).

\begin{tabular}{|c|c|c|c|c|c|c|c|c|c|c|c|c|c|c|c|c|}
\hline$n$ & & & & & & & & & $\lambda \mu)$ & & & & & & & \\
\hline 28 & $102)$ & & & & & & & & & & & & & & & \\
\hline 26 & $(04)$ & (12) & (20) & & & & & & & & & & & & & \\
\hline 24 & (06) & (14) & $(22)^{2}$ & (11) & $(00)$ & & & & & & & & & & & \\
\hline 22 & (08) & (16) & $(24)^{2}$ & (32) & (40) & (13) & (21) & $(02)^{2}$ & & & & & & & & \\
\hline 20 & & (18) & $(26)^{2}$ & (34) & $(42)^{2}$ & (15) & (23) & $(04)^{2}$ & (31) & $(20)^{2}$ & (12) & & & & & \\
\hline 18 & & (28) & (36) & $(44)^{2}$ & (17) & (25) & $(06)^{2}$ & (52) & (60) & (33) & (41) & (14) & $(22)^{3} \quad(11)$ & $(00)$ & & \\
\hline 16 & & & (46) & (27) & (54) & $(62)^{2}$ & (35) & (43) & $(24)^{3}$ & (16) & (08) & (51) & $(40)^{2} \quad(32)$ & (13) & (21) & $(02)^{2}$ \\
\hline 14 & & & (64) & (72) & (45) & $(26)^{2}$ & (53) & (34) & $(42)^{3}$ & (61) & (80) & (15) & $(01)^{2} \quad(23)$ & (31) & (12) & $(20)^{2}$ \\
\hline 12 & & (82) & (63) & $(44)^{2}$ & (71) & (52) & $(60)^{2}$ & (25) & (06) & (33) & (14) & (41) & $(22)^{3} \quad(11)$ & (00) & & \\
\hline 10 & & (81) & $(62)^{z}$ & (43) & $(24)^{2}$ & (51) & (32) & $(40)^{2}$ & (13) & $(02)^{2}$ & (21) & & & & & \\
\hline 8 & $(80)$ & (61) & $(42)^{2}$ & (23) & (04) & (31) & (12) & $(20)^{2}$ & & & & & & & & \\
\hline 6 & $(60)$ & (41) & $(22)^{2}$ & (11) & (00) & & & & & & & & & & & \\
\hline 4 & $(40)$ & (21) & (02) & & & & & & & & & & & & & \\
\hline 2 & $(20)$ & & & & & & & & & & & & & & & \\
\hline
\end{tabular}

of a boson mapping procedure ${ }^{20}$ ); but the more cumbersome boson mapping techniques of ref. ${ }^{20}$ ) would be difficult to generalize to states with $u \neq 0$.

\subsection{STATES WITH $w=0$}

In this case $\omega_{i}=n_{i}$; all $K^{2}$ submatrices are 1-dimensional, and the $K^{2}$ ratio of eq. (24) becomes

$$
\frac{K^{2}\left(\left[n^{\prime}\right]\right)}{K^{2}(\lfloor n])}=\frac{2}{3} \Omega-n_{i}+i-1
$$


where $n_{i}^{\prime}=n_{i}+2$; ( $i$ gives the index of the tableau row to which the two squares are added). This leads at once to

$$
\begin{aligned}
& K^{2}\left(\left[n_{1} n_{2} n_{3}\right]\right)=\frac{\left(\frac{2}{3} \Omega+2\right) ! !\left(\frac{2}{3} \Omega+1\right) ! !\left(\frac{2}{3} \Omega\right) ! !}{\left(\frac{2}{3} \Omega-n_{3}+2\right) ! !\left(\frac{2}{3} \Omega-n_{2}+1\right) ! !\left(\frac{2}{3} \Omega-n_{1}\right) ! !}, \\
& \left\langle u=0\left[\boldsymbol{n}^{\prime}\right]\left\|\boldsymbol{A}^{+}\right\| u=0[\boldsymbol{n}]\right\rangle=\sqrt{\left(\frac{2}{3} \Omega-n_{i}+i-1\right)}\left\langle\left[\boldsymbol{n}^{\prime}\right]\|z\|[\boldsymbol{n}]\right\rangle .
\end{aligned}
$$

Note also that the factor $\frac{2}{3} \Omega-n_{i}+i-1$ would be replaced with $\frac{2}{3} \Omega-\frac{2}{3} u-n_{i}+i-1$ in states with $u=3,6 \ldots$ but $\left(\lambda_{\sigma} \mu_{\sigma}\right)=(00)$. The maximum possible $n_{1}$ values are $\frac{2}{3}(\Omega-u)$.

\subsection{STATES WITH $w=1$}

In this case $[\sigma]=[100],\left(\lambda_{\sigma} \mu_{\sigma}\right)=(10)$. The possible $[\omega]$ are $\left[n_{1}+1 n_{2} n_{3}\right],\left[n_{1} n_{2}+\right.$ $\left.1 n_{3}\right],\left[n_{1} n_{2} n_{3}+1\right]$ each with the unique $[n]$ value of $\left[n_{1} n_{2} n_{3}\right]$. (Recall that the $n_{i}$ are all even). Eqs. (24) and (20) at once lead to

$$
\left(K^{2}([\omega])\right)_{[n][n]}=\frac{\left(\frac{2}{3} \Omega+2\right) ! !\left(\frac{2}{3} \Omega+1\right) ! !\left(\frac{2}{3} \Omega-2\right) ! !}{\left(\frac{2}{3} \Omega-n_{3}+2-\Delta_{3}\right) ! !\left(\frac{2}{3} \Omega-n_{2}+1-\Delta_{2}\right) ! !\left(\frac{2}{3} \Omega-n_{1}-\Delta_{1}\right) ! !},
$$

where

$$
\Delta_{j}=2 \quad \text { if } \omega_{j}=n_{j}+1,
$$

$$
\Delta_{j}=0 \quad \text { for the other two factors. }
$$

Now

$\left\langle[[1] \times[\boldsymbol{n}]]\left[\omega^{\prime}\right]\left\|\boldsymbol{A}^{\dagger}\right\|[[1] \times[\overline{\boldsymbol{n}}]][\omega]\right\rangle$

$$
=\left[\Lambda_{[n]\left[\omega^{\prime}\right]}-\Lambda_{[\bar{n}][\omega]}\right]^{1 / 2} U\left((10)\left(\lambda_{\bar{n}} \mu_{\bar{n}}\right)\left(\lambda_{\omega^{\prime}} \mu_{\omega^{\prime}}\right)(20) ;\left(\lambda_{\omega} \mu_{\omega}\right) ;\left(\lambda_{n} \mu_{n}\right)\right) \times\langle[[n]\|z\|[\bar{n}]\rangle,
$$

where $\left(\lambda_{\omega^{\prime}} \mu_{\omega^{\prime}}\right)=\left(\omega_{1}^{\prime}-\omega_{2}^{\prime}, \omega_{2}^{\prime}-\omega_{3}^{\prime}\right)$, e.g.; and where there are in general 5 possible [n], $[\omega]$ combinations for each [ $\left.\omega^{\prime}\right]$. For convenience, the possible $\Lambda_{[n]\left[\omega^{\prime}\right]}-A_{[\bar{n}][\omega]}$ for the $u=1$ states are given in table 5 .

\subsection{STATES WITH $u=2,\left(\lambda_{\sigma} \mu_{\sigma}\right)=(01)$}

In this case, with $[\sigma]=[11]$, there are again only three possible $[\omega]$, with $[\omega]=$ $\left[n_{1}+1 n_{2}+1 n_{3}\right],\left[n_{1}+1 n_{2} n_{3}+1\right]$, or $\left[n_{1} n_{2}+1 n_{3}+1\right]$. Since the $n_{i}$ must all be even the $[n]$-values are uniquely determined by $[\omega]$. Eqs. (24) and (20) now lead to

$$
\left(K^{2}([\omega])\right)_{[n][n]}=\frac{\left(\frac{2}{3} \Omega+2\right) ! !\left(\frac{2}{3} \Omega-1\right) ! !\left(\frac{2}{3} \Omega-2\right) ! !}{\left(\frac{2}{3} \Omega-n_{3}+2-\Delta_{3}\right) ! !\left(\frac{2}{3} \Omega-n_{2}+1-\Delta_{2}\right) ! !\left(\frac{2}{3} \Omega-n_{1}-\Delta_{1}\right) ! !},
$$

where

$$
\begin{array}{ll}
\Delta_{j}=2 & \text { if } \omega_{j}=n_{j}+1 \\
\Delta_{j}=0 & \text { for the third factor } .
\end{array}
$$


3.4. STATES WITH $u=2,\left(\lambda_{\sigma} \mu_{\sigma}\right)=(20)$

With $[\sigma]=[200]$, there are two basic types of U(3) representations. First, for $[\omega]$ with two odd values of $\omega_{j},[n]$ is uniquely determined by $[\omega]$ and this case leads to 1-dimensional $K^{2}$ matrices.

With $[n]=\left[n_{1} n_{2} n_{3}\right]$ and $[\omega]=\left[n_{1}+1 n_{2}+1 n_{3}\right],\left[n_{3}+1 n_{2} n_{3}+1\right]$, or $\left[n_{1} n_{2}+1 n_{3}+\right.$ 1]:

$$
\left(K^{2}([\omega])\right)_{[n][n]}=\frac{\left(\frac{2}{3} \Omega+2\right) ! !\left(\frac{2}{3} \Omega-1\right) ! !\left(\frac{2}{3} \Omega-4\right) ! !\left(\frac{2}{3} \Omega\right)}{\left(\frac{2}{-2} \Omega-n_{3}+2-\Delta_{3}\right) ! !\left(\frac{2}{3} \Omega-n_{2}+1-\Delta_{2}\right) ! !\left(\frac{2}{3} \Omega-n_{1}-\Delta_{1}\right) ! !}
$$

where

$$
\begin{array}{ll}
\Delta j=2 & \text { if } \omega_{j}=n_{j}+1 \\
\Delta_{j}=0 & \text { for the third factor . }
\end{array}
$$

For the second type of $[\omega]$, with all $\omega_{i}$ even, $[\boldsymbol{n}]$ can, in general, have three possible values, and the most general $K K^{\dagger}$ submatrices for this type of $[\omega]$ will be $3 \times 3$ matrices. (Note: Since many of these $3 \times 3$ matrices will have zero eigenvalues, we will use the forms given by eqs. (27) and (28) for $K^{-1}$ and $K$ and will therefore not use the language $K^{2}$ ). In this case, eqs. (22) will lead to a system of linear equations for the $K K^{\dagger}$ matrix elements for [ $\left.\omega^{\prime}\right]$ in terms of known $K K^{\dagger}$ matrix elements for $[\omega]$ with $\sum_{i} \omega_{i}=\left(\sum_{i} \omega_{i}^{\prime}\right)-2$. The needed SU(3) $U$-coefficients can be read in analytic form from table 5 of ref. ${ }^{21}$ ) with the use of eq. (18) of ref. ${ }^{21}$ ). Some of the calculational details for this case will be given in appendix A. The final results are:

With $\omega_{i}$ all even, and the common factor defined by

$$
[\text { C.F. }] \equiv \frac{\left(\frac{2}{3} \Omega+2\right) ! !\left(\frac{2}{3} \Omega-1\right) ! !\left(\frac{2}{3} \Omega-4\right) ! !}{\left(\frac{2}{3} \Omega-\omega_{3}+2\right) ! !\left(\frac{2}{3} \Omega-\omega_{2}+1\right) ! !\left(\frac{2}{3} \Omega-\omega_{1}\right) ! !},
$$

TABLE 5

The $\left(\boldsymbol{A}_{[n]\left[\omega^{\prime}\right]}-\boldsymbol{A}_{[n][\omega]}\right)$ factors for $u=1$ states

\begin{tabular}{cccc}
\hline$\left[\omega^{\prime}\right]$ & {$[\omega]$} & {$[\bar{n}]$} & $\left(A_{[n]\left[\omega^{\prime}\right]}-A_{[\bar{n}[[\omega]}\right)$ \\
\hline$\left[n_{1}+1 n_{2} n_{3}\right]$ & {$\left[n_{1}-1 n_{2} n_{3}\right]$} & {$\left[n_{1}-2 n_{2} n_{3}\right]$} & $\left(\frac{2}{3} \Omega-n_{1}\right)$ \\
& {$\left[n_{1}+1 n_{2}-2 n_{3}\right]$} & {$\left[n_{1} n_{2}-2 n_{3}\right]$} & $\left(\frac{2}{3} \Omega-n_{2}+3\right)$ \\
& {$\left[n_{1}+1 n_{2} n_{3}-2\right]$} & {$\left[n_{1} n_{2} n_{3}-2\right]$} & $\left(\frac{2}{3} \Omega-n_{3}+4\right)$ \\
& {$\left[n_{1} n_{2}-1 n_{3}\right]$} & {$\left[n_{1} n_{2}-2 n_{3}\right]$} & $\left(\frac{2}{3} \Omega-n_{1}\right)$ \\
& {$\left[n_{1} n_{2} n_{3}-1\right]$} & {$\left[n_{1} n_{2} n_{3}-2\right]$} & $\left(\frac{2}{3} \Omega-n_{1}\right)$ \\
{$\left[n_{1} n_{2}+1 n_{3}\right]$} & {$\left[n_{1}-2 n_{2}+1 n_{3}\right]$} & {$\left[n_{1}-2 n_{2} n_{3}\right]$} & $\left(\frac{2}{3} \Omega-n_{1}+2\right)$ \\
& {$\left[n_{1} n_{2}-1 n_{3}\right]$} & {$\left[n_{1} n_{2}-2 n_{3}\right]$} & $\left(\frac{2}{3} \Omega-n_{2}+1\right)$ \\
& {$\left[n_{1} n_{2}+1 n_{3}-2\right]$} & {$\left[n_{1} n_{2} n_{3}-2\right]$} & $\left(\frac{2}{3} \Omega-n_{3}+4\right)$ \\
& {$\left[n_{1}-1 n_{2} n_{3}\right]$} & {$\left[n_{1}-2 n_{2} n_{3}\right]$} & $\left(\frac{2}{3} \Omega n_{2} 1\right)$ \\
& {$\left[n_{1} n_{2} n_{3}-1\right]$} & {$\left[n_{2} n_{2} n_{3}-2\right]$} & $\left(\frac{2}{3} \Omega-n_{2}+1\right)$ \\
{$\left[n_{1} n_{2} n_{3}+1\right]$} & {$\left[n_{1}-2 n_{2} n_{3}+1\right]$} & {$\left[n_{1}-2 n_{2} n_{3}\right]$} & $\left(\frac{2}{3} \Omega-n_{1}+2\right)$ \\
& {$\left[n_{1} n_{2}-2 n_{3}+1\right]$} & {$\left[n_{1} n_{2}-2 n_{3}\right]$} & $\left(\frac{2}{3} \Omega-n_{2}+3\right)$ \\
& {$\left[n_{1} n_{2} n_{3}-1\right]$} & {$\left[n_{1} n_{2} n_{3}-2\right]$} & $\left(\frac{2}{3} \Omega-n_{3}+2\right)$ \\
& {$\left[n_{1}-1 n_{2} n_{3}\right]$} & {$\left[n_{1}-2 n_{2} n_{3}\right]$} & $\left(\frac{2}{3} \Omega-n_{3}+2\right)$ \\
& {$\left[n_{1} n_{2}-1 n_{3}\right]$} & {$\left[n_{1} n_{2}-2 n_{3}\right]$} & $\left(\frac{2}{3} \Omega-n_{3}+2\right)$ \\
\hline
\end{tabular}


$\left(K K^{\dagger}\left(\left[\omega_{1} \omega_{2} \omega_{3}\right]\right)\right)_{\left[\omega_{1}-2 \omega_{2} \omega_{3}\right]\left[\omega_{1} \omega_{2}-2 \omega_{3}\right]}$

$$
=-[\text { C.F. }] \times \frac{\sqrt{\left(\omega_{1}+2\right)\left(\omega_{2}+1\right)\left(\omega_{1}-\omega_{2}\right)\left(\omega_{1}-\omega_{2}+2\right)\left(\omega_{1}-\omega_{3}+1\right)\left(\omega_{2}-\omega_{3}\right)}}{\left(\omega_{1}-\omega_{2}+1\right) \sqrt{\left(\omega_{1}-\omega_{3}+2\right)\left(\omega_{2}-\omega_{3}+1\right)}},
$$

$\left(K K^{\dagger}\left(\left[\omega_{1} \omega_{2} \omega_{3}\right]\right)\right)_{\left[\omega_{1}-2 \omega_{2} \omega_{3}\right]\left[\omega_{1} \omega_{2} \omega_{3}-2\right]}$

$$
=-[\text { C.F. }] \times \frac{\sqrt{\left(\omega_{1}+2\right) \omega_{3}\left(\omega_{1}-\omega_{2}\right)\left(\omega_{1}-\omega_{3}+1\right)\left(\omega_{1}-\omega_{3}+3\right)\left(\omega_{2}-\omega_{3}+2\right)}}{\left(\omega_{1}-\omega_{3}+2\right) \sqrt{\left(\omega_{1}-\omega_{2}+1\right)\left(\omega_{2}-\omega_{3}+1\right)}},
$$

$\left(K K^{\dagger}\left(\left[\omega_{1} \omega_{2} \omega_{3}\right]\right)\right)_{\left[\omega_{1} \omega_{2}-2 \omega_{3}\right]\left[\omega_{1} \omega_{2} \omega_{3}-2\right]}$

$$
=-[\text { C.F. }] \times \frac{\sqrt{\left(\omega_{2}+1\right) \omega_{3}\left(\omega_{1}-\omega_{2}+2\right)\left(\omega_{1}-\omega_{3}+3\right)\left(\omega_{2}-\omega_{3}\right)\left(\omega_{2}-\omega_{3}+2\right)}}{\left(\omega_{2}-\omega_{3}+1\right) \sqrt{\left(\omega_{1}-\omega_{2}+1\right)\left(\omega_{1}-\omega_{3}+2\right)}},
$$

$\left(K K^{\dagger}\left(\left[\omega_{1} \omega_{2} \omega_{3}\right]\right)_{\left[\omega_{1}-2 \omega_{2} \omega_{3}\right]\left[\omega_{1}-2 \omega_{2} \omega_{3}\right]}\right.$

$$
=[\text { C.F. }] \times\left[\frac{2}{3} \Omega\left(\frac{2}{3} \Omega-\omega_{1}+1\right)-\frac{\left(\omega_{1}+2\right)\left(\omega_{1}-\omega_{2}\right)\left(\omega_{1}-\omega_{3}+1\right)}{\left(\omega_{1}-\omega_{2}+1\right)\left(\omega_{1}-\omega_{3}+2\right)}\right],
$$

$\left(K K^{\dagger}\left(\left[\omega_{1} \omega_{2} \omega_{3}\right]\right)\right)_{\left[\omega_{1} \omega_{2}-2 \omega_{3}\right]\left[\omega_{1} \omega_{2}-2 \omega_{3}\right]}$

$$
=[\mathrm{C} . \mathrm{F} .] \times\left[\frac{2}{3} \Omega\left(\frac{2}{3} \Omega-\omega_{2}+2\right)-\frac{\left(\omega_{2}+1\right)\left(\omega_{1}-\omega_{2}+2\right)\left(\omega_{2}-\omega_{3}\right)}{\left(\omega_{1}-\omega_{2}+1\right)\left(\omega_{2}-\omega_{3}+1\right)}\right],
$$

$\left(K K^{\dagger}\left(\left[\omega_{1} \omega_{2} \omega_{3}\right]\right)\right)_{\left[\omega_{1} \omega_{2} \omega_{3}-2\right]\left[\omega_{1} \omega_{2} \omega_{3}-2\right]}$

$$
=[\text { C.F. }] \times\left[\frac{2}{3} \Omega\left(\frac{2}{3} \Omega-\omega_{3}+3\right)-\frac{\omega_{3}\left(\omega_{1}-\omega_{3}+3\right)\left(\omega_{2}-\omega_{3}+2\right)}{\left(\omega_{1}-\omega_{3}+2\right)\left(\omega_{2}-\omega_{3}+1\right)}\right] .
$$

These $3 \times 3$ matrices reduce to simpler matrices in many cases. Since these special cases are often cases with zero eigenvalues it is important to examine them in some detail in order to determine the Pauli-allowed domain of the $\operatorname{Sp}(6)$ irrep $\left(\frac{1}{3} \Omega \frac{1}{3} \Omega \frac{1}{3} \Omega-\right.$ 2).

(i) With $\omega_{2}=\omega_{3}=0$ the $K K^{\dagger}$-matrix for $[\omega]=\left[\omega_{1} 00\right]$ is a $1 \times 1$ matrix with

$$
K K^{\dagger}=\frac{\left(\frac{2}{3} \Omega-4\right) ! !}{\left(\frac{2}{3} \Omega-\omega_{1}-2\right) ! !}
$$

In this case the highest possible $\omega_{1}$ is $\left(\frac{2}{3} \Omega-2\right)$, (not $\frac{2}{3} \Omega$ as in the $u=0$ case).

(ii) With $\omega_{3}=0$ the $K K^{\dagger}$ matrix for $[\omega]=\left[\omega_{1} \omega_{2} 0\right]$ is a $2 \times 2$-matrix, in general with two nonzero eigenvalues. In the special case with $\omega_{1}=\frac{2}{3} \Omega$ this $2 \times 2$ matrix has the form

$$
\begin{aligned}
K K^{\dagger}= & \frac{[\mathrm{C} . \mathrm{F} .]\left(\omega_{1}=\frac{2}{3} \Omega, \omega_{3}=0\right)}{\left(\omega_{1}-\omega_{2}+1\right)} \\
& \times\left(\begin{array}{cc}
\omega_{2} & -\sqrt{\omega_{2}\left(\omega_{1}+1\right)\left(\omega_{1}-\omega_{2}\right)\left(\omega_{1}-\omega_{2}+2\right)} \\
-\sqrt{\omega_{2}\left(\omega_{1}+1\right)\left(\omega_{1}-\omega_{2}\right)\left(\omega_{1}-\omega_{2}+2\right)} & \left(\omega_{1}+1\right)\left(\omega_{1}-\omega_{2}\right)\left(\omega_{1}-\omega_{2}+2\right)
\end{array}\right) .
\end{aligned}
$$


It can be seen at once that the determinant of this matrix is zero. One of the eigenvalues is zero, and there is only one Pauli-allowed state of the type $\left[\omega_{1} \omega_{2} 0\right]$ with $\omega_{1}=\frac{2}{3} \Omega$, corresponding to a specific linear combination of states with $[\boldsymbol{n}]=$ $\left[\omega_{1}-2 \omega_{2} 0\right]$ and $\left[\omega_{1} \omega_{2} 20\right]$. This explains why states with $n=12,(\lambda \mu)=(82) ; n-14$, $(\lambda \mu)=(64)$ in table 4 are simple rather than 2 -fold.

(iii) With $\omega_{2}=\omega_{3}$ the $K K^{\dagger}$ matrix for $[\omega]=\left[\omega_{1} \omega_{2} \omega_{2}\right]$ is again a $2 \times 2$ matrix with two possible $[\boldsymbol{n}]=\left[\omega_{1}-2 \omega_{2} \omega_{2}\right]$ or $\left[\omega_{1} \omega_{2} \omega_{2}-2\right]$, in general. In the additional special case with $\omega_{1}=\frac{2}{3} \Omega$ this $2 \times 2$ matrix has the form

$$
\begin{aligned}
K K^{\dagger} & =\frac{[\text { C.F. }]\left(\omega_{1}=\frac{2}{3} \Omega, \omega_{3}=\omega_{2}\right)}{\left(\omega_{1}-\omega_{2}+2\right)} \\
& \times\left(\begin{array}{cc}
2 \omega_{2} & -\sqrt{2 \omega_{2}\left(\omega_{1}+2\right)\left(\omega_{1}-\omega_{2}\right)\left(\omega_{1}-\omega_{2}+3\right)} \\
-\sqrt{2 \omega_{2}\left(\omega_{1}+2\right)\left(\omega_{1}-\omega_{2}\right)\left(\omega_{1}-\omega_{2}+3\right)} & \left(\omega_{1}+2\right)\left(\omega_{1}-\omega_{2}\right)\left(\omega_{1}-\omega_{2}+3\right)
\end{array}\right) .
\end{aligned}
$$

This again has zero determinant and hence only one nonzero eigenvalue and thus only a single Pauli-allowed state. This explains why the state with $n=14,(\lambda \mu)=(80)$ in table 4 is simple rather than two-fold.

For particle numbers $n>\Omega$, matrix elements are determined most readily from particle-hole conjugation, using the states with $n=2 \Omega-u$ as the vector "vacuum" states and polynomials $Z(\boldsymbol{A})$ in the pair annihilation operators for the state construction. However, this is not necessary. States with $n=20$ and $(\lambda \mu)=(42)$ in table 4 , e.g., could be constructed through the $3 \times 3 K K^{\dagger}$ matrix for $[\omega]=\left[\begin{array}{lll}10 & 6\end{array}\right]$. This has the numerical value

$$
K K^{*}=\frac{12 \cdot 10 \cdot 9 \cdot 7 \cdot 6 \cdot 4 \cdot 2}{5} \times\left(\begin{array}{ccc}
8 & -14 \sqrt{6} & -6 \sqrt{35} \\
-14 \sqrt{6} & 272 & -2 \sqrt{210} \\
-6 \sqrt{35} & -2 \sqrt{210} & 420
\end{array}\right)
$$

Again, the determinant is zero. This $K K^{\dagger}$ matrix has only two nonzero eigenvalues. There are only two Pauli-allowed states with $n=20(\lambda \mu)=(42)$ in agreement with the findings for $n=10(\lambda \mu)=(24)$.

\section{Concluding remarks}

If the $k$-active $\mathrm{Sp}(6) \supset \mathrm{U}(3)$ branch of the favored S,D-pair algebra yields an approximately good symmetry for nuclei in certain mass regions, then states of low generalized S,D-pair seniority may be of greatest relevance for the low-lying states of such nuclei. The state construction of such low-seniority states has been given in very general and explicit form via vector coherent state techniques. States of even higher seniority may be constructed by the same techniques, although it may be necessary to treat specific cases numerically. For $u=3$, e.g., with $\left(\lambda_{\sigma} \mu_{\sigma}\right)=(30),(11)$, 
or (00), it may not be worthwhile to derive general algebraic formulae. For $\left(\lambda_{\sigma} \mu_{\sigma}\right)=$ (11) multiplicity labels, $\rho$, begin to come into play and general algebraic expressions become cumbersome.

With the results given in sect. 3 it is straightforward to evaluate matrix elements of fermion hamiltonians of the type used in the interacting boson model, i.e. hamiltonians built from the group generators themselves. Moreover, such hamiltonians can be treated on an equal footing for both even and odd nuclei. Positions of higher seniority states can easily be determined, and with somewhat generalized hamiltonians an assessment could be made of the effects of higher-seniority admixtures into the dominant low-seniority states.

\section{Appendix A}

The method of calculation of the $3 \times 3 K^{\dagger}$ submatrices for general [ $\omega$ ], with all $\omega_{i}$ even integers, for the $u=2$ case with $[\sigma]=[200]$ will be sketched in this appendix. The basic equation for the recursive determination of these matrix elements is eq. (22), where $\left[\omega^{\prime}\right]$ is such that $\sum_{i} \omega_{i}^{\prime}=\sum_{i} \omega_{i}+2$. By choosing $[\omega]=\left[n_{1}+1 n_{2}+1 n_{3}\right]$ and the fixed $\left[n^{\prime}\right]=\left[n_{1}+2 n_{2} n_{3}\right]$ or $\left[n_{1} n_{2}+2 n_{3}\right]$ for $\left[\omega^{\prime}\right]=\left[n_{1}+2 n_{2}+2 n_{3}\right]$, two equations are obtained for the unknown $K K^{\dagger}\left(\left[\omega^{\prime}\right]\right)$ in terms of the known quantities given by eq. (38). Altogether this type of choice for the fixed representations $[\omega]$, $\left[\omega^{\prime}\right]$ and $\left[n^{\prime}\right]$ leads to six equations. With the shorthand notation

$$
\begin{aligned}
& \left.\left(K K^{\dagger}\left(\omega_{1}^{\prime} \omega_{2}^{\prime} \omega_{3}^{\prime}\right]\right)\right)_{\left[\omega_{1}^{\prime}-2 \omega_{2}^{\prime} \omega_{3}^{\prime}\right]\left[\omega_{1}^{\prime}-2 \omega_{2}^{\prime} \omega_{3}^{\prime}\right]} \equiv\left(K K^{\dagger}\right)_{11}, \\
& \left(K K^{\dagger}\left(\left[\omega_{1}^{\prime} \omega_{2}^{\prime} \omega_{3}^{\prime}\right]\right)\right)_{\left[\omega_{1}-2 \omega_{2}^{\prime} \omega_{3}^{\prime}\right]\left[\omega_{1} \omega_{2}^{\prime}-2 \omega_{3}^{\prime}\right]} \equiv\left(K K^{\dagger}\right)_{12}, \text { etc. },
\end{aligned}
$$

these six equations are

$$
\begin{aligned}
& \left(K K^{\dagger}\right)_{11}-x\left(K K^{\dagger}\right)_{12}=\left(\frac{2}{3} \Omega-\omega_{1}^{\prime}+1\right) K_{0}^{2}, \\
& \left(K K^{\dagger}\right)_{22}-\frac{1}{x}\left(K K^{\dagger}\right)_{12}=\left({ }_{3}^{2} \Omega-\omega_{2}^{\prime}+2\right) K_{0}^{2}, \\
& \left(K K^{\dagger}\right)_{11}-y\left(K K^{\dagger}\right)_{13}=\left(\frac{2}{3} \Omega-\omega_{1}^{\prime}+1\right) K_{0}^{2}, \\
& \left(K K^{\dagger}\right)_{33}-\frac{1}{y}\left(K K^{\dagger}\right)_{13}=\left(\frac{2}{3} \Omega-\omega_{3}^{\prime}+3\right) K_{0}^{2}, \\
& \left(K K^{\dagger}\right)_{22}-z\left(K K^{\dagger}\right)_{23}=\left(\frac{2}{3} \Omega-\omega_{2}^{\prime}+2\right) K_{0}^{2}, \\
& \left(K K^{\dagger}\right)_{33}-\frac{1}{z}\left(K K^{\dagger}\right)_{23}=\left(\frac{2}{3} \Omega-\omega_{3}^{\prime}+3\right) K_{0}^{2},
\end{aligned}
$$

where

$$
K_{0}^{2}=\frac{\left(\frac{2}{3} \Omega+2\right) ! !\left(\frac{2}{3} \Omega-1\right) ! !\left(\frac{2}{3} \Omega-4\right) ! !\left(\frac{2}{3} \Omega\right)}{\left(\frac{2}{3} \Omega-\omega_{3}^{\prime}+2\right) ! !\left(\frac{2}{3} \Omega-\omega_{2}^{\prime}+1\right) ! !\left(\frac{2}{3} \Omega-\omega_{1}\right) ! !},
$$


and

$$
\begin{aligned}
& x=\sqrt{\frac{\left(\omega_{1}^{\prime}+2\right)\left(\omega_{1}^{\prime}-\omega_{2}^{\prime}\right)\left(\omega_{1}^{\prime}-\omega_{3}^{\prime}+1\right)\left(\omega_{2}^{\prime}-\omega_{3}^{\prime}+1\right)}{\left(\omega_{2}^{\prime}+1\right)\left(\omega_{1}^{\prime}-\omega_{2}^{\prime}+2\right)\left(\omega_{1}^{\prime}-\omega_{3}^{\prime}+2\right)\left(\omega_{2}^{\prime}-\omega_{3}^{\prime}\right)}}, \\
& z=\sqrt{\frac{\left(\omega_{2}^{\prime}+1\right)\left(\omega_{1}^{\prime}-\omega_{2}^{\prime}+2\right)\left(\omega_{1}^{\prime}-\omega_{3}^{\prime}+2\right)\left(\omega_{2}^{\prime}-\omega_{3}^{\prime}\right)}{\omega_{3}^{\prime}\left(\omega_{1}^{\prime}-\omega_{2}^{\prime}+1\right)\left(\omega_{1}^{\prime}-\omega_{3}^{\prime}+3\right)\left(\omega_{2}^{\prime}-\omega_{3}^{\prime}+2\right)}}, \\
& y=x z .
\end{aligned}
$$

Because of the last relation the six equations (A.1) are not independent, and these are therefore not sufficient to solve for the six unknowns $\left(K K^{\dagger}\right)_{i j}$. Eq. (22) can, however, also be used to get a simple recursion equation for matrix elements such as $\left(K K^{\dagger}\right)_{23}$

$$
\begin{aligned}
& \frac{\left(K K^{\dagger}\left(\left[\omega_{1}^{\prime} \omega_{2}^{\prime} \omega_{3}^{\prime}\right]\right)\right)_{\left[\omega_{1}^{\prime} \omega_{2}^{\prime}-2 \omega_{3}^{\prime}\right]\left[\omega_{1}^{\prime} \omega_{2}^{\prime} \omega_{3}^{\prime}-2\right]}}{\left(K K^{\dagger}\left(\left[\omega_{1}^{\prime} \omega_{2}^{\prime} \omega_{3}^{\prime}-2\right]\right)\right)_{\left[\omega_{1}^{\prime} \omega_{2}^{\prime}-2 \omega_{3}^{\prime}-2\right]\left[\omega_{1}^{\prime} \omega_{2}^{\prime} \omega_{3}^{\prime}-4\right]}} \\
& \quad=\left(\frac{2}{3} \Omega-\omega_{3}^{\prime}+4\right) \frac{\left(\omega_{2}^{\prime}-\omega_{3}^{\prime}+3\right)}{\left(\omega_{2}^{\prime}-\omega_{3}^{\prime}+1\right)} \sqrt{\frac{\omega_{3}^{\prime}\left(\omega_{1}^{\prime}-\omega_{3}^{\prime}+3\right)\left(\omega_{1}^{\prime}-\omega_{3}^{\prime}+4\right)\left(\omega_{2}^{\prime}-\omega_{3}^{\prime}\right)}{\left(\omega_{3}^{\prime}-2\right)\left(\omega_{1}^{\prime}-\omega_{3}^{\prime}+2\right)\left(\omega_{1}^{\prime}-\omega_{3}^{\prime}+5\right)\left(\omega_{2}^{\prime}-\omega_{3}^{\prime}+4\right)}} .
\end{aligned}
$$

This can be used to relate $\left(K K^{\dagger}\right)_{23}$ to the specific $\left(K K^{\dagger}\left(\left[\omega_{1}^{\prime} \omega_{2}^{\prime} 2\right]\right)\right)_{23}$, which can be simply related to $K K^{\dagger}\left(\left[\omega_{1}^{\prime} \omega_{2}^{\prime}\right]\right)$ matrix elements with two-rowed [ $\left.\omega^{\prime}\right]$. The matrix elements of the latter can be determined from the first two of eqs. (A.1) with $\omega_{3}^{\prime}=0$, and the simple recursion formula

$$
\begin{aligned}
& \frac{\left(K K^{\dagger}\left(\left[\omega_{1}^{\prime} \omega_{2}^{\prime}\right]\right)\right)_{\left[\omega_{1}^{\prime}-2 \omega_{2}^{\prime}\right]\left[\omega_{2}^{\prime} \omega_{2}^{\prime}-2\right]}}{\left(K K^{\dagger}\left(\left[\omega_{1}^{\prime} \omega_{2}^{\prime}-2\right]\right)\right)_{\left[\omega_{1}^{\prime}-2 \omega_{2}^{\prime}-2\right]\left[\omega_{i}^{\prime} \omega_{2}^{\prime}-4\right]}} \\
& \quad=\left(\frac{2}{3} \Omega-\omega_{2}^{\prime}+3\right) \frac{\left(\omega_{1}^{\prime}-\omega_{2}^{\prime}+3\right)}{\left(\omega_{1}^{\prime}-\omega_{2}^{\prime}+1\right)} \sqrt{\frac{\omega_{2}^{\prime}\left(\omega_{1}^{\prime}-\omega_{2}^{\prime}\right)}{\left(\omega_{2}^{\prime}-2\right)\left(\omega_{1}^{\prime}-\omega_{2}^{\prime}+4\right)}} .
\end{aligned}
$$

\section{References}

1) J.N. Ginocchio, Ann. of Phys. 126 (1980) 234

2) Cheng-Li Wu, Da Hsuan Feng, Xuan-Gen Chen, Jin-Quan Chen and M.W. Guidry, Phys. Lett. B168 (1986) 313

3) Jin-Quan Chen, Da Hsuan Feng and Cheng-Li Wu, Phys. Rev. C34 (1986) 2269

4. Cheng-Li Wu, Da Hsuan Feng, Xuan-Gen Chen, Jin-Quan Chen and M.W. Guidry, Phys. Rev. C36 (1987) 1157

5) R.F. Casten, Cheng-Li Wu, Da Hsuan Feng, J.N. Ginocchio and Xiao-Ling Han, Phys. Rev. Lett. 56 (1986) 2578

6) Zi-Min Lu, Xin-Wan Pan, Jin-Quan Chen, Xuan-Gen Chen and Da IIsuan Feng, 1987 Preprint

7) K.T. Hecht, Nucl. Phys. A475 (1987) 276

8) D.J. Rowe, J. Math. Phys. 25 (1984) 2662

9) D.J. Rowe, G. Rosensteel and R. Gilmore, J. Mat. Phys. 26 (1985) 2787

10) J. Deenen and C. Quesne, J. Math. Phys. 25 (1984) 1638, 2354

11) C. Quesne, J. Math. Phys. 27 (1986) 428 and 869

12. K.T. Hecht, The vector coherent state method and its application to problems of higher symmetries, Lecture Notes in Physics 290 (Springer, Heidelberg, 1987) 
13) J.P. Draayer and Yorck Leschber, Branching rules for unitary symmetries of the nuclear shell model (1987), Louisiana State University publication

14) D.J. Rowe, Rep. Prog. Phys. 48 (1985) 1419

15) Y. Suzuki and K.T. Hecht, Nucl. Phys. A455 (1986) 315

16) Y. Suzuki, Nucl. Phys. A470 (1987) 119

17) K.T. Hecht, Proc. of the VIIIth Oaxtepec Symp. on Nuclear physics, Notas de Física, vol. 8, no. 1, p. 165 (1985)

18) D.J. Rowe, B.G. Wybourne and P. Butler, J. Phys. A18 (1985) 939

19) J.P. Draayer and Y. Akiyama, J. Math. Phys. 14 (1973) 1904, and Comput. Phys. Commun. 5 (1973) 405

20) D. Bonatsos, A. Klein and Quing-Ying Zhang, Phys. Rev. C34 (1986) 686;

D. Bonatsos and A. Klein, Phys. Rev. C31 (1985) 992

21) K.T. Hecht, Nucl. Phys. 62 (1965) I 